\title{
Magnetofection based on superparamagnetic iron oxide nanoparticle-mediated low IncRNA HOTAIR expression decreases the proliferation and invasion of glioma stem cells
}

\author{
KAN FANG $^{1 *}$, PEIFENG LIU ${ }^{1 *}$, SUYAN DONG $^{1}$, YANJIE GUO $^{1}$, XINXIN CUI $^{1}$, XIAOYING ZHU ${ }^{1}$, \\ XUAN LI ${ }^{1}$, LIANGHAN JIANG ${ }^{5 *}$, TE LIU ${ }^{2-4}$ and YUNCHENG WU ${ }^{1}$ \\ ${ }^{1}$ Department of Neurology, Shanghai General Hospital, Shanghai Jiao Tong University School of Medicine, Shanghai 200080; \\ ${ }^{2}$ Shanghai Geriatric Institute of Chinese Medicine, Longhua Hospital, Shanghai University of Traditional Chinese Medicine, \\ Shanghai 200031, P.R. China; ${ }^{3}$ Department of Pathology, Yale University School of Medicine, New Haven, \\ CT 06520, USA; ${ }^{4}$ Shanghai Tenth People's Hospital, Medical School, Tongji University, Shanghai 200072; \\ ${ }^{5}$ Shanghai Mental Health Center, Shanghai Jiao Tong University School of Medicine, Shanghai 200030, P.R. China
}

Received April 11, 2016; Accepted May 27, 2016

DOI: 10.3892/ijo.2016.3571

\begin{abstract}
Glioma stem cells (GSCs) are a special subpopulation of glioma cells that are key to the sensitivity of tumors to treatments and to the possibility of tumor recurrence. Identifying new strategies that inhibit the growth of GSCs are therefore important for developing novel therapies for glioblastoma multiforme (GBM). In this study, $\mathrm{CD} 133^{+}$human glioma stem cells were isolated and cultured. Magnetic nanoparticles were used to mediate the expression of siRNAs targeting the HOTAIR (si-HOTAIR) sequence in human gliomas. Effect of downregulation of HOTAIR expression on proliferation, invasion and in vivo tumorigenicity of human GSCs and underlying molecular mechanisms were further evaluated. The results of the MTT assay and flow cytometric analysis showed that downregulation of HOTAIR expression inhibited cell proliferation and induced cell cycle arrest. Transwell assays demonstrated that downregulation of HOTAIR expression resulted in a decrease in the invasive capability of GSCs. Moreover, magnetic nanoparticle-mediated low expression of HOTAIR effectively reduced the tumorigenic capacity of glioma stem cells in vivo. In addition, the results of qRT-PCR and western blot analysis demonstrated that downregulation
\end{abstract}

Correspondence to: Professor Te Liu, Shanghai Geriatric Institute of Chinese Medicine, Longhua Hospital, Shanghai University of Traditional Chinese Medicine, Shanghai 200031, P.R. China E-mail: teliu79@126.com

Professor Yuncheng Wu, Department of Neurology, Shanghai General Hospital, Shanghai Jiao Tong University School of Medicine, 100 HaiNing Road, Shanghai 200080, P.R. China

E-mail: yunchw@medmail.com.cn

${ }^{*}$ Contributed equally

Key words: glioma stem cells, magnetofection based on superparamagnetic iron oxide nanoparticles, HOTAIR, PDCD4, proliferation and invasion of HOTAIR expression significantly increased the expression of PDCD4 in GSCs, in addition to reducing the expression of CCND1 and CDK4. An in-depth mechanistic analysis showed that downregulation of HOTAIR expression reduced the recruitment of downstream molecules, EZH2 and LSD1, thereby activating the expression of PDCD4 at the transcriptional level. In conclusion, downregulation of HOTAIR expression effectively promoted the expression of PDCD4, thereby inhibiting the proliferation, invasion and in vivo tumorigenicity of human GSCs.

\section{Introduction}

Recent studies have shown that a very small percentage of cells $(0.1-1 \%)$ present in a variety of solid tumors possess characteristics similar to stem cells, such stem-like tumor cells have been designated cancer stem cells (CSCs) by cancer researchers. CSCs are a critical factor in the sensitivity of tumors to treatments and tumor recurrence (1). Glioma is the most common type of primary central nervous system (CNS) tumor. The isolation and identification of glioma stem cells are achieved mainly by virtue of functional distinctions and molecular markers. Currently recognized markers specific for glioma stem cells include CD133, integrin $\alpha 6$, CD171, CD15 and nestin (2). Among these markers, CD133 is the most widely recognized marker for glioma stem cells. The subset of glioma cells expressing high levels of CD133 can be isolated using flow cytometry-based cell sorting techniques and serially passaged in a serum-free culture system, allowing investigation of the functional status of these cells in vitro and the development of therapeutic strategies specifically targeting such cells (3).

Long non-coding RNAs (lncRNAs) are a class of noncoding RNA transcripts with a length of $>200$ nucleotides. They regulate gene expression at various levels in the form of RNA (4). HOX transcript antisense RNA (HOTAIR) was the first trans-acting lncRNA gene to be discovered. The expression of HOTAIR is upregulated in a variety of tumor 
cells, which is related to poor prognosis of the tumors $(5,6)$. The 5 ' terminus of HOTAIR binds to chromatin-modifying complexes such as polycomb repressive complex 2 (PRC2) which mediates the trimethylation of lysine 27 on histone $\mathrm{H} 3$ (H3K27), thereby silencing the transcription of specific genes. EZH2 plays a major role in the trimethylation of H3K27 (5). The 3' terminus of HOTAIR binds to the histone deacetylase 1 (HDAC1)/lysine-specific demethylase 1 (LSD1)/ REST corepressor 1 (CoREST)/RE1-silencing transcription factor (REST) repressor complex. The complex mediates the demethylation of dimethylated histone H3 lysine 4 (H3K4me2), thereby silencing the transcription of target genes $(7,8)$. In a previous study, we showed that interference with HOTAIR expression inhibits the proliferation and in vitro tumorigenicity of renal cancer cells (9). Research has also demonstrated that interference with HOTAIR inhibits the cell cycle progression and invasive capability of glioma cells; however, adenoviral vectors were used in that study, which lack clinical applicability (10). In this study, a non-viral carrier was used, which further allowed the possibility of employing HOTAIR as a molecular target to achieve effective treatment in vivo.

Magnetic nanomaterials are a class of nanomaterials that generally refers to materials with a grain size of $<100 \mathrm{~nm}$, consisting of iron, cobalt, nickel and their alloys. Among a series of magnetic nanoparticles, iron oxide nanoparticles have been widely studied due to their high saturation magnetization value, low toxicity, easily obtainable raw material and high surface reactivity. In recent years, iron oxide nanoparticles have received increasing attention as gene carriers. When the size of a magnetic nanoparticle is $<20 \mathrm{~nm}$, it often exhibits superparamagnetic properties. Superparamagnetic iron oxide nanoparticles (SPIONs) have currently become a hotspot of gene carrier research because of their controllable properties, high stability and susceptibility to modification. After binding to plasmid DNA or small interfering RNA (siRNA), SPIONs deliver the bound nucleic acids into mammalian cells under the influence of an external magnetic field. SPIONs overcome the intracellular and extracellular barriers impeding gene delivery via magnetic absorption, thereby increasing the local DNA concentration and improving transfection efficiency (11-13).

Based on the findings described above, a HOTAIR siRNA sequence was constructed in this study. In addition, SPIONs were used to mediate the transfection and expression of siRNAs targeting HOTAIR in CD133+ GSCs. The goal of this study was to evaluate whether si-HOTAIR is capable of reducing the proliferation, invasion and tumorigenicity of GSCs through targeting the expression of the programmed cell death 4 (PDCD4).

\section{Materials and methods}

Isolation and culture of $\mathrm{CD} 133^{+}$human glioma stem cells . $\mathrm{CD}_{133^{+}}$human glioma stem cells were isolated from the human glioma cell line SHG44 (Shanghai Institutes for Biological Sciences, Chinese Academy of Sciences). Briefly, after digestion with $0.25 \%$ trypsin, the detached SHG44 cells were collected via centrifugation and then resuspended in $0.5 \mathrm{ml}$ of ice-cold sterile phosphate-buffered saline (PBS, Hyclone). Subsequently, $5 \mu \mathrm{l}$ of a fluorescein isothiocyanate (FITC)-conjugated anti-human CD133 antibody (eBioscience) was added to the cell suspension to achieve a final concentration of $0.01 \mathrm{mg} / \mathrm{ml}$. The cells were then incubated for $30 \mathrm{~min}$ at $4^{\circ} \mathrm{C}$ in the dark. Upon the completion of antigen-antibody interactions, the cells were washed twice with ice-cold PBS. Then, the isolation and enrichment of $\mathrm{CD} 133^{+}$human glioma stem cells were achieved using a flow cytometer (BD FACS Aria, BD Bioscience, CA, USA). CD133 ${ }^{+}$human glioma stem cells were subsequently seeded at a density of 1,000 cells $/ \mathrm{ml}$ in Dulbecco's modified Eagle's medium (DMEM)-F12 medium (Hyclone) containing $10 \mathrm{ng} / \mathrm{ml}$ basic fibroblast growth factor (bFGF), $10 \mathrm{ng} / \mathrm{ml}$ epidermal growth factor (EGF), $5 \mu \mathrm{g} / \mathrm{ml}$ insulin and $0.5 \%$ bovine serum albumin (BSA) (all from Sigma-Aldrich, St. Louis, MO, USA) to generate nonadherent spherical clusters. The cells were cultured until passage 3 .

SPION-induced transfection of microRNA plasmid DNA into cells. SPIONs were purchased from Novobio (Novobio Biotechnology Co., Ltd., Shanghai, China). According to the procedure specified in the instruction manual and the methods described in previous studies, $5 \mu 1$ of SPIONs $(0.2 \mathrm{mM})$ was mixed thoroughly with $5 \mu \mathrm{l}$ of a HOTAIR-siRNA or mocksiRNA plasmid $(10 \mu \mathrm{M})$. The mixture was then vortexed for $10 \mathrm{sec}$ and allowed to stand at room temperature (RT) for $20 \mathrm{~min}$. Subsequently, the SPION-siRNA plasmid DNA conjugates $(10 \mu \mathrm{l})$ were mixed with $90 \mu \mathrm{l}$ of serum-free DMEM and added to the cells $\left(1 \times 10^{4}\right.$ cells $\left./ \mathrm{ml}\right)$, and the cells were cultured in the presence of the SPION-siRNA plasmid DNA conjugates for $72 \mathrm{~h}$ at $37^{\circ} \mathrm{C}$ in an atmosphere of $5 \% \mathrm{CO}_{2}$.

CCK-8 assay. Briefly, various groups of cells were seeded into $96-$ well cell culture plates at a density of $2 \times 10^{3}$ cells $/ \mathrm{ml}$. At $72 \mathrm{~h}$ after transfection, $10 \mu \mathrm{l}$ of CCK-8 solution (Sigma-Aldrich Chemical) was added to each group of cells. The cells were then incubated at $37^{\circ} \mathrm{C}$ for $3 \mathrm{~h}$, after which the cell culture plates were placed on a microplate reader, and the absorbance at a wavelength of $450 \mathrm{~nm}$ was recorded. The cell proliferation inhibition rate (\%) was calculated using the following formula: (1 - OD value of cells in the experimental group - blank / OD value of cells in the control group - blank) x $100 \%$.

RNA extraction and analysis via quantitative real-time PCR ( $q R T-P C R)$. Total RNA was extracted from each group of cells using the TRIzol reagent (Invitrogen) in accordance with the manufacturer's instructions. Total RNA was treated with DNAse I (Sigma-Aldrich), quantified and then reverse transcribed into complementary DNA (cDNA) using the ReverTra Ace- $\alpha$ First-Strand cDNA Synthesis kit (Toyobo). qRT-PCR was performed on the RealPlex4 real-time PCR detection system (Eppendorf Co. Ltd., Germany), and SYBR Green Real-Time PCR Master Mix (Toyobo) was used as the fluorescent dye to label the amplified nucleic acids. qRT-PCR amplification was performed over 40 cycles with denaturation at $95^{\circ} \mathrm{C}$ for $15 \mathrm{sec}$ annealing at $58^{\circ} \mathrm{C}$ for $45 \mathrm{sec}$. Target cDNA was quantified using the relative quantification method. A comparative threshold cycle $(\mathrm{Ct})$ was used to determine gene expression relative to a control (calibrator) and steady-state mRNA levels are reported as an n-fold difference relative to the calibrator. For each sample, the maker genes $\mathrm{Ct}$ values were normalized using the formula $\Delta \mathrm{Ct}=\mathrm{Ct}$ _markers - 
Table I. The sequences of qRT-PCR primers.

\begin{tabular}{ll}
\hline Gene product & Forward (F) and reverse (R) primers \\
\hline 18 s rRNA & F: CAGCCACCCGAGATTGAGCA \\
R: TAGTAGCGACGGGCGGTGTG \\
Fd GA & R: CACGTGGAATACACCTGCAA \\
Fd133 & F: CCATTGGCATTCTCTTTGAA \\
& R: TTTGGATTCATATGCCTTCTGT \\
Nestin & F: AAGATGTCCCTCAGCCTGG \\
Bdnf & R: GAGGGAAGTCTTGGAGCCAC \\
& F: GTCTCTGGGGATGCAGAG \\
Pdcd4 & F: GGCCTTCATGCAACCAAAGT \\
& R: TTCATCACCGGAAAAGAGAGA \\
Ccnd1 & F: TCCTCTCCAAAATGCCAGAG \\
& R: GGCGGATTGGAAATGAACTT \\
Fdk4 & F: TGCAGTCCACATATGCAACA \\
& R: GTCGGCTTCAGAGTTTCCAC \\
\end{tabular}

Ct_18 sRNA. To determine the relative expression levels, the following formula was used $\Delta \Delta \mathrm{Ct}=\Delta \mathrm{Ct}$ _si-HOTAIR - $\Delta \mathrm{Ct}$ _Mock. The values used to plot relative expressions of markers were calculated using the expression $2-\Delta \Delta \mathrm{Ct}$. The mRNA levels were calibrated based on levels of $18 \mathrm{sec}$ RNA. The cDNA of each gene was amplified with primers as previously described (Table I).

PI stain and FCM assay. PI staining and flow cytometric (FCM) assays were performed according to procedures that have been described previously $(14,15)$. Briefly, cells $\left(5 \times 10^{5}\right.$ cells $\left./ \mathrm{ml}\right)$ were harvested and fixed in $1 \mathrm{ml}$ of ice-cold $70 \%$ ethanol for $48 \mathrm{~h}$. After fixation, the cells were centrifuged at $1,500 \mathrm{r} / \mathrm{min}$ and $4^{\circ} \mathrm{C}$ for $5 \mathrm{~min}$, and the cell pellets were collected. Subsequently, the cells were incubated in PI staining solution (Sigma Chemicals) for $30 \mathrm{~min}$ at $4{ }^{\circ} \mathrm{C}$ in the dark. After PI staining, the cell cycle distribution was examined in each group of cells via flow cytometry (BD FACSAria), and the results were analyzed using CellQuest software.

Transwell migration assay. Briefly, cells were seeded into the upper chambers of Transwell plates (membrane pore size: $8.0 \mu \mathrm{m})$ at a density of $2 \times 10^{3}$ cells $/ \mathrm{ml}$ in $200 \mu \mathrm{l}$ of serum-free cell culture medium. A $600-\mu 1$ volume of complete medium containing $10 \%$ fetal bovine serum (FBS) was added to the lower chambers of the Transwell plates. The cells were then cultured for $48 \mathrm{~h}$ at $37^{\circ} \mathrm{C}$ in an atmosphere of $5 \% \mathrm{CO}_{2}$. Subsequently, cells attached to the lower surface of membrane were fixed with $4 \%$ paraformaldehyde at RT for $30 \mathrm{~min}$ and stained with 4,6-diamidino-2-phenylindole (DAPI, SigmaAldrich Chemical) for $10 \mathrm{~min}$. Three non-overlapping fields of view were selected under a microscope, and the total number of cells was counted.

Northern blotting. Northern blot analysis was performed according to a procedure described previously $(14,15)$. Briefly, total RNA was extracted from all groups of cells using the TRIzol kit and then analyzed and quantified. Next, $20 \mu \mathrm{g}$ of high-quality total RNA was subjected to gel electrophoresis on a 7.5 M urea-12\% formaldehyde [polyacrylamide (PAA)] denaturing gel. After electrophoresis, the RNA was transferred to Hybond $\mathrm{N}^{+}$nylon membranes (Amersham, Freiburg, Germany) and then cross-linked to the membranes via exposure to ultraviolet (UV) radiation for $30 \mathrm{sec}$ (UV dose:1,200 $\mathrm{mJ} / \mathrm{cm}^{2}$ ). To examine the expression status of miR-374a, a labeled antisense DNA probe against miR-374a was allowed to hybridize with the immobilized RNA. After hybridization and extensive washing of the membranes, the membranes were exposed to Kodak XAR-5 film (Sigma-Aldrich Chemical) for 20-40 h. As a positive control, the human U6 small nuclear RNA (snRNA) probe (5'-GCAGGGGCCATGCTAATCTTCTCTGTATCG-3') was hybridized to the RNA on the membranes. The exposure time for the U6 snRNA probe was controlled between 15 and $30 \mathrm{~min}$.

Western blotting. Western blot analysis was performed according to a procedure described previously $(14,15)$. Briefly, total protein was extracted from each group of cells and subjected to sodium dodecyl sulfate polyacrylamide gel electrophoresis (SDS-PAGE) on a $12 \%$ denaturing gel. After electrophoresis, total protein was transferred to a polyvinylidene difluoride (PVDF) membrane (Millipore). The membrane was blocked, washed and then incubated with primary antibodies at $37^{\circ} \mathrm{C}$ for $45 \mathrm{~min}$ (Table II). After extensive washing, the membrane was incubated with secondary antibodies at $37^{\circ} \mathrm{C}$ for $45 \mathrm{~min}$, followed by washing 4 times for $14 \mathrm{~min}$ each with Tris-buffered saline-Tween-20 (TBST) at RT. The blot was developed with the enhanced chemiluminescence (ECL) kit (Pierce Biotechnology) and film exposed to light (Sigma-Aldrich Chemical) for visualization.

CHIP. The antibodies used in the experiments included an anti-trimethylated H3K27 antibody, anti-dimethylated H3K4 antibody and normal rabbit IgG (negative control). Briefly, the cells were fixed with $1 \%$ paraformaldehyde at $37^{\circ} \mathrm{C}$ for $30 \mathrm{~min}$ and then incubated with $125 \mathrm{mM}$ glycine at RT for $10 \mathrm{~min}$ to terminate the cross-linking reaction. Subsequently, the cells were sonicated on ice until the DNA had been sheared into chromatin fragments of $200-1,000 \mathrm{bp}$ in size. The DNA fragments were then incubated with primary antibodies at $4^{\circ} \mathrm{C}$ overnight. Immunoprecipitates were ultimately obtained via adsorption to protein $\mathrm{A} / \mathrm{G}$ plus-agarose beads and subjected to PCR amplification. The PCR conditions were as follows: denaturation at $95^{\circ} \mathrm{C}$ for $30 \mathrm{sec}$; annealing at $55^{\circ} \mathrm{C}$ for $30 \mathrm{sec}$; elongation at $72^{\circ} \mathrm{C}$ for $30 \mathrm{sec}$; and a total of 33 amplification cycles. The amplification products were examined via agarose gel electrophoresis.

In vivo xenograft experiments. The experiments were conducted according to a procedure described previously $(14,15)$. Briefly, various groups of cells were transfected with different plasmids. Logarithmically growing cells $\left(1 \times 10^{5}\right.$ cells $/ \mathrm{ml}$ ) were collected and inoculated subcutaneously into BALB/C $\mathrm{C}^{\text {nu/nu }}$ mice. Each experimental group contained 4 mice (6-8-week-old male BALB/ $\mathrm{C}^{\mathrm{nu} / \mathrm{nu}}$ mice, provided by the Experimental Animal Center of Fudan University). Animal 
Table II. Primary antibodies, their source and dilutions.

\begin{tabular}{llc}
\hline Antibodies & \multicolumn{1}{c}{ Companies } & Applications \\
\hline Rabbit anti-human PDCD4 (\#9535) & Cell Signaling Technology, Inc. (Danvers, MA, USA) & IF $(1: 100)$ \\
Rabbit anti-human BDNF (\#3897) & Cell Signaling Technology, Inc. (Danvers, MA, USA) & WB $(1: 1,000)$ \\
& & IF $(1: 100)$ \\
WB $(1: 1,000)$ \\
Rabbit anti-human CCND1 (\#2978) & Cell Signaling Technology, Inc. (Danvers, MA, USA) & WB $(1: 1,000)$ \\
Rabbit anti-human CDK4 (\#12790) & Cell Signaling Technology, Inc. (Danvers, MA, USA) & WB $(1: 1,000)$ \\
Rabbit anti-human Ki67 (\#9129) & Cell Signaling Technology, Inc. (Danvers, MA, USA) & IF $(1: 100)$ \\
Rabbit anti-human H3K4Me2 (\#9725) & Cell Signaling Technology, Inc. (Danvers, MA, USA) & IP $(1: 100)$ \\
Rabbit anti-human H3K4Me3 (\#9751) & Cell Signaling Technology, Inc. (Danvers, MA, USA) & IP $(1: 100)$ \\
Rabbit anti-human EZH2 (\#5246) & Cell Signaling Technology, Inc. (Danvers, MA, USA) & IP $(1: 100)$ \\
Rabbit anti-human LSD1 (\#2184) & Cell Signaling Technology, Inc. (Danvers, MA, USA) & IP $(1: 100)$ \\
Rabbit anti-human GAPDH (\#5174) & Cell Signaling Technology, Inc. (Danvers, MA, USA) & WB $(1: 1,000)$ \\
\hline
\end{tabular}

experiments were performed in accordance with the National Institutes of Health Guide for the Care and Use of Laboratory Animals (NIH publication no. 80-23) and approved by the ethics committee for the use of experimental animals in Fudan University and every attempt was made to limit animal numbers and suffering. After 8 weeks of continuous monitoring, the mice were sacrificed, and their tumors were removed. The tumors were subsequently weighed, and the tumor volume was calculated according to the following formula: tumor volume $\left(\mathrm{mm}^{3}\right)=\left(\mathrm{ab}^{2}\right) / 2$ [a, the longest axis $(\mathrm{mm}) ; \mathrm{b}$, the shortest axis $(\mathrm{mm})]$.

Histopathology assay. Briefly, fresh tissues were fixed via immersion in 4\% paraformaldehyde (Sigma-Aldrich) at RT for $30 \mathrm{~min}$. The tissues were then subjected to gradient ethanol dehydration, embedded in paraffin, sectioned (thickness, $6 \mu \mathrm{m})$, and dewaxed in xylene. Tissue sections were stained with hematoxylin and eosin (H\&E, Sigma-Aldrich), permeabilized with xylene (Sigma-Aldrich) and mounted in neutral resin (Sigma-Aldrich).

Immunohistochemical staining assay. Briefly, fresh tissues were fixed via immersion in $4 \%$ paraformaldehyde (Sigma-Aldrich) at RT for $30 \mathrm{~min}$. The tissues were then subjected to gradient ethanol dehydration, embedded in paraffin, sectioned (thickness, $6 \mu \mathrm{m}$ ), and dewaxed in xylene. Next, the tissue sections were blocked at $37^{\circ} \mathrm{C}$ for $30 \mathrm{~min}$ in immunohistochemistry (IHC) blocking solution (Beyotime Biotechnology Co., Ltd., Zhejiang, China). After removal of the blocking solution, the tissue sections were washed 3 times at RT for 5 min each using IHC wash buffer (Beyotime Biotechnology Co., Ltd.). Subsequently, the tissue sections were incubated with primary antibodies (Table II) at $37^{\circ} \mathrm{C}$ for $45 \mathrm{~min}$. After removal of the primary antibodies, the tissue sections were washed 3 times at RT for 5 min each using IHC wash buffer (Beyotime Biotechnology Co., Ltd.). The tissue sections were then incubated with secondary antibodies (Table I) at $37^{\circ} \mathrm{C}$ for $45 \mathrm{~min}$. The secondary antibodies were subsequently discarded, and the tissue sections were washed 3 additional times at RT for 5 min each using IHC wash buffer (Beyotime Biotechnology Co., Ltd.). Finally, the tissue sections were mounted using neutral resin (Sigma-Aldrich) or fluorescence mounting medium (Sigma-Aldrich).

Transmission electron microscopy (TEM) analysis. Tissue samples were fixed and embedded according to the procedure described in a previous study. The tissue samples were first fixed with $1 \%$ glutaraldehyde (Sigma-Aldrich) for $4 \mathrm{~h}$ and then with $1 \%$ osmium tetroxide (Sigma-Aldrich) for $1 \mathrm{~h}$. Subsequently, the tissue samples were dehydrated in acetone and embedded in resin 12 (Ted Pella, USA). Ultrathin sections of the embedded tissues (cross-sectional thickness, $70 \mathrm{~nm}$ ) were attached to a copper grid, stained with $1 \%$ uranyl acetate (Sigma-Aldrich) and 1\% lead citrate (Sigma-Aldrich), and imaged using a JEM-1230 transmission electron microscope (Jeol, Japan).

Statistical analysis. Each experiment was performed as least three times, and data are presented as the mean \pm SE where applicable. Differences were evaluated using Student's t-test, and a probability of $<0.05$ was considered to be statistically significant.

\section{Results}

$\mathrm{CD} 133^{+}$human glioma stem cells express a high level of HOTAIR. The $\mathrm{CD}_{133}{ }^{+}$cell subset $(1.07 \pm 0.21 \%)$ was isolated using a flow cytometry-based cell sorting technique (Fig. 1A). It was observed that the $\mathrm{CD} 133^{+}$cells grew in suspension. The cells were densely packed, and intercellular spaces were not clearly detectable. The colonies of CD133 ${ }^{+}$cells appeared full and highly refractive (Fig. 1B). Moreover, the $\mathrm{CD}_{133^{+}}$cell subset exhibited a significantly higher proliferation rate. It reached the maximum growth rate after $\sim 4$ days in suspension culture (Fig. 1C). qRT-PCR revealed that the mRNA levels of CD133, CD44, nestin and brain-derived neurotrophic factor (Bdnf) were significantly higher in the subset of CD133+ cells compared with the control group (Fig. 1D). The above experiments demonstrated that the subset of $\mathrm{CD}_{13} 3^{+}$cells was indeed glioma stem cells. Northern blot analysis results 

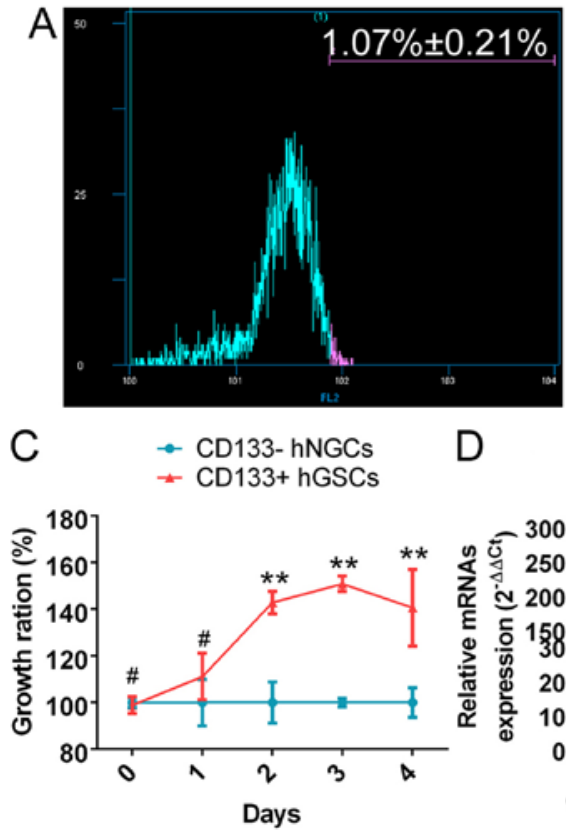

D

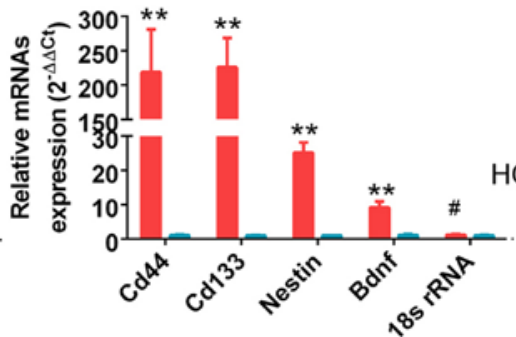
CD133- hNGCs
CD133+ hGSCs

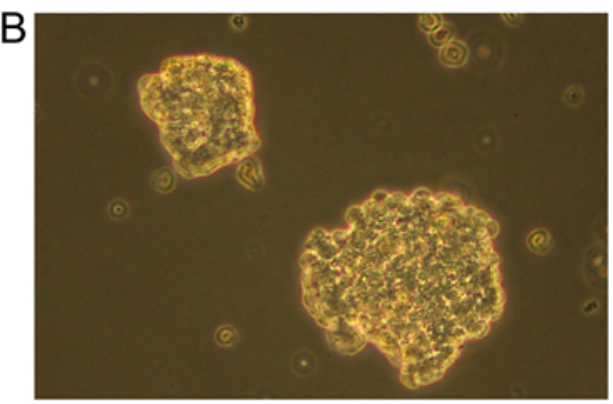

$\mathrm{E}$

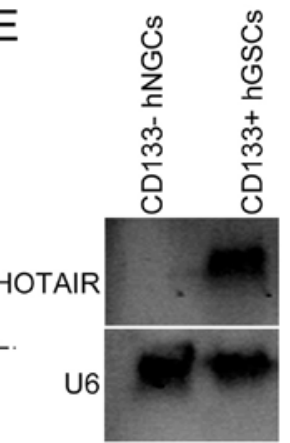

Figure 1. Human glioma stem cells express high levels of HOTAIR. (A) CD133 ${ }^{+}$human glioma stem cells were isolated from glioma tissues using a flow cytometry-based cell sorting technique. (B) In a serum-free culture system, CD133 ${ }^{+}$human glioma stem cells grew clonally in suspension (original magnification, x200). (C) The results of the CCK-8 assay indicated that CD133+ human glioma stem cells possessed a significantly greater proliferative capability compared with CD133- human glioma cells. (D) The results of qRT-PCR revealed that the expression of the neural stem cell-related markers CD44, CD133, nestin and Bdnf was significantly elevated in $\mathrm{CD} 133^{+}$human glioma stem cells compared with CD133- human glioma cells. (E) The results of northern blot analysis demonstrated that the intensity of the HOTAIR hybridization signal was significantly stronger in CD133+ human glioma stem cells in comparison with CD133- human glioma cells. ${ }^{*} \mathrm{P}<0.05, \mathrm{n}=3$.
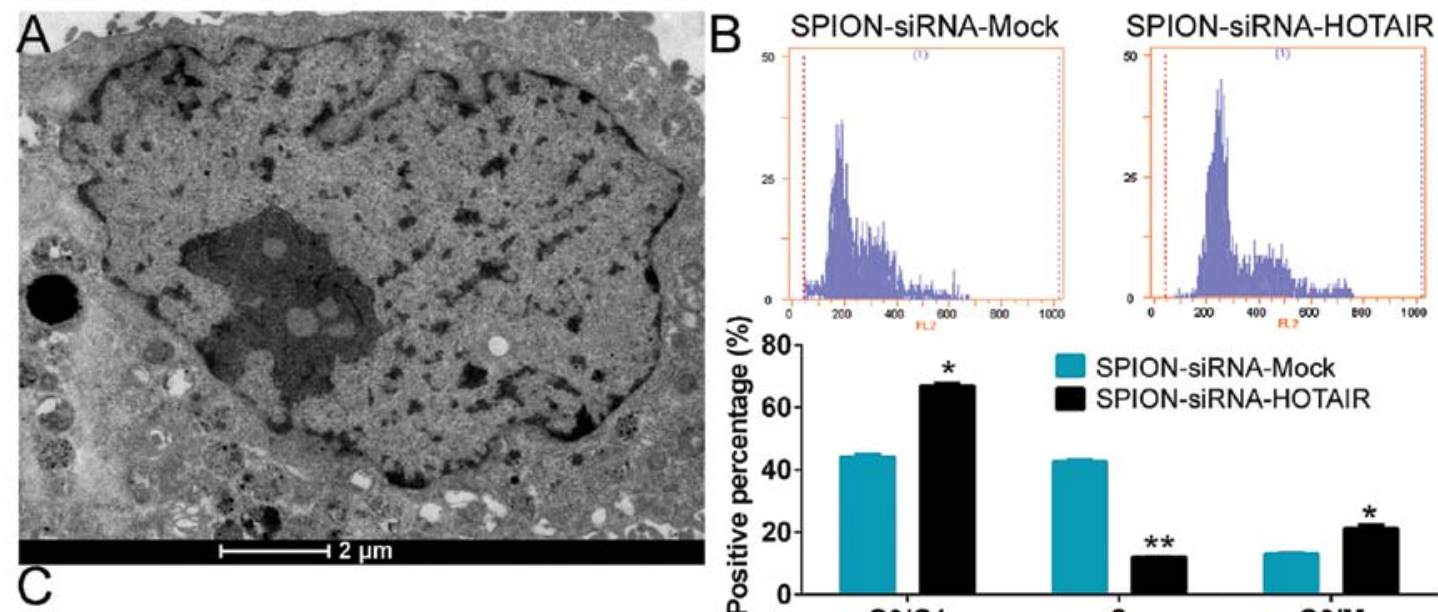

SPION-SIRNA-Mock

SPION-siRNA-HOTAIR

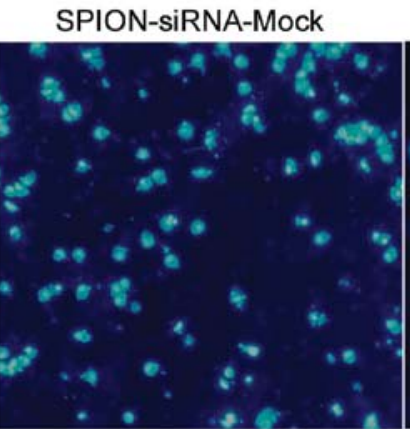
SPION-sIRNA-HOTAIR
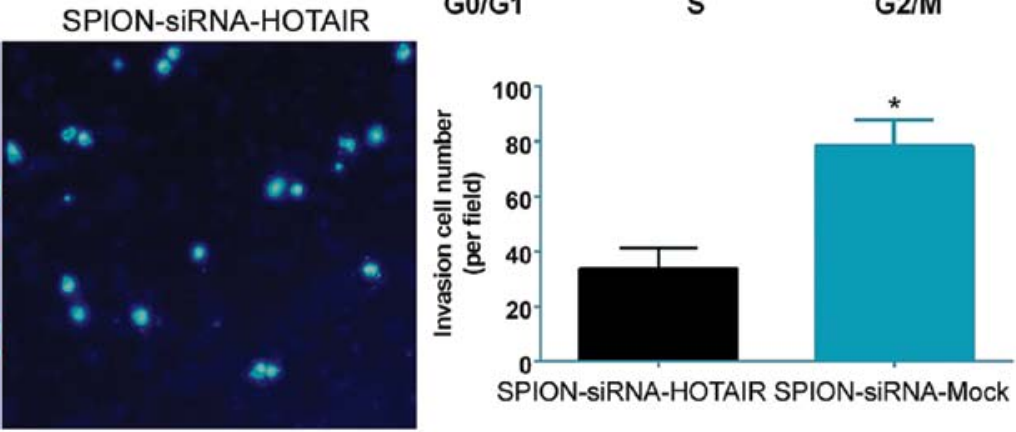

Figure 2. Downregulation of HOTAIR expression significantly inhibits the proliferation and invasion of CD133 $3^{+}$human glioma stem cells. (A) TEM revealed that multiple high-density electron clouds were present in the group of cells subjected to SPION-mediated si-HOTAIR transfection. The results indicated that SPION successfully delivered si-HOTAIR into the cells. (B) Flow cytometric analysis demonstrated that compared with the control group, a considerably higher percentage of $\mathrm{CD}_{133^{+}}$human glioma stem cells transfected with SPION-si-HOTAIR conjugates were in the G0/G1 and G2/M phases of the cell cycle. In contrast, the percentage of S-phase cells was significantly lower in the group of CD133 ${ }^{+}$human glioma stem cells subjected to SPION-mediated si-HOTAIR transfection compared with the control group. (C) The results of Transwell assays showed that the number of invading cells was significantly lower in the group subjected to SPION-mediated si-HOTAIR transfection compared with the control group. "P<0.05, n=3. 


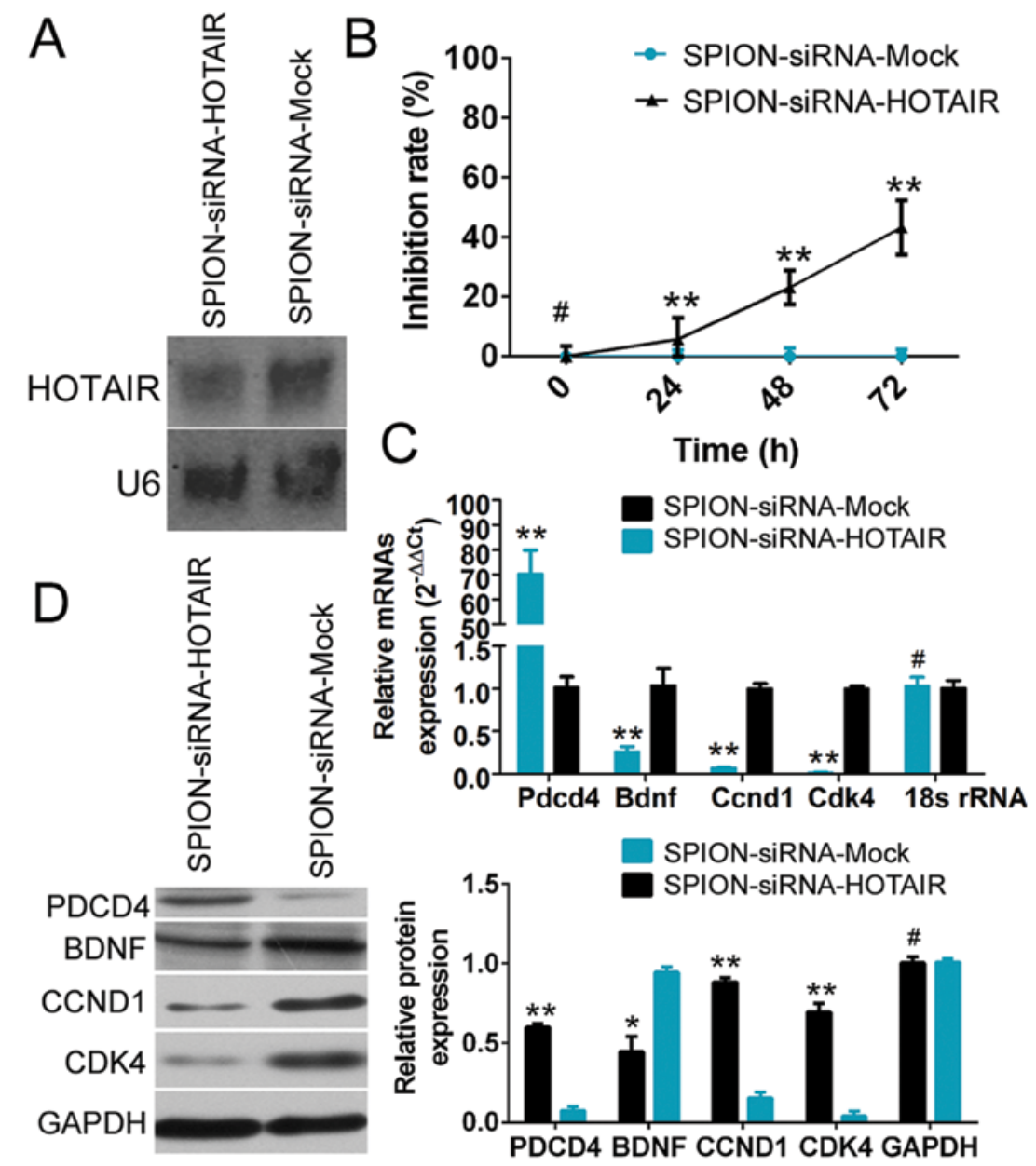

Figure 3. Downregulation of HOTAIR expression significantly inhibits the expression of PDCD4 and cell cycle regulatory proteins in human glioma stem cells. (A) Northern blot analysis showed that the hybridization signal yielded by the HOTAIR probe was significantly lower in CD133+ human glioma stem cells transfected with SPION/si-HOTAIR conjugates in comparison with the control group. (B) The CCK-8 assay showed that the cell proliferation inhibition rate was significantly higher in the group of $\mathrm{CD}_{133^{+}}$human glioma stem cells subjected to SPION-mediated si-HOTAIR transfection compared with the control group. (C) The results of qRT-PCR showed that compared with the control group, the expression level of PDCD4 mRNA was significantly higher, while the expression levels of Bdnf, CCND1 and CDK4 mRNAs were considerably lower in the group of CD133 ${ }^{+}$human glioma stem cells subjected to SPION-mediated si-HOTAIR transfection. (D) The results of western blot analysis showed that compared with the control group, the expression level of the PDCD4 protein was significantly higher, while the expression levels of the Bdnf, CCND1 and CDK4 proteins were considerably lower in the group of CD133 ${ }^{+}$human glioma stem cells subjected to SPION-mediated si-HOTAIR transfection. ${ }^{*} \mathrm{P}<0.05,{ }^{* *} \mathrm{P}<0.01,{ }^{*} \mathrm{P}>0.05, \mathrm{n}=3$.

revealed a significantly stronger endogenous HOTAIR hybridization signal in $\mathrm{CD} 133^{+}$human glioma stem cells compared with normal CD133- glioma cells (Fig. 1E).

SPION-mediated si-HOTAIR transfection of human glioma stem cells inhibits cell proliferation and invasion. Si-HOTAIR was added to cultured $\mathrm{CD} 133^{+}$human glioma stem cells after being crosslinked to SPION. The glioma stem cells were observed under a projection electron microscope at $72 \mathrm{~h}$. Multiple circular-shaped, dense electron clouds of $\sim 80-100 \mathrm{~nm}$ in size were detected in both the cytoplasm and the nucleus of the glioma stem cells, indicating that SPION successfully entered the cells (Fig. 2A). Flow cytometric analysis of the cell cycle showed that SPION-mediated si-HOTAIR transfection markedly reduced the number of human glioma stem cells in the S phase $(12.06 \pm 0.08 \%)$ of the cell cycle, while significantly increasing the number of cells in both the G0/G1 phase $(66.78 \pm 0.96 \%)$ and the G2/M phase $(21.17 \pm 1.04 \%$, Fig. $2 B)$. In addition, Transwell assays found that the number of cells invading through the Transwell insert membrane was signifi- cantly lower in the group that was nanomagnetically transfected with si-HOTAIR compared with the group subjected to SPION-mediated transfection of the si-mock control (Fig. 2C). The above results demonstrated that SPION effectively mediated the entry of exogenous si-RNA sequences into the cells and that interference with HOTAIR expression effectively suppressed the proliferation and invasion of $\mathrm{CD} 133^{+}$human glioma stem cells in vitro.

Si-HOTAIR-mediated interference with HOTAIR expression in human glioma stem cells promotes the expression of PDCD4 and inhibits the expression of cell cycle regulatory proteins. Northern blot analysis showed that the HOTAIR probe yielded a significantly weaker hybridization signal in si-HOTAIR-transfected human glioma stem cells compared with the si-mock-transfected group (Fig. 3A). The CCK-8 cell proliferation assay demonstrated that SPION-mediated low HOTAIR expression in $\mathrm{CD} 133^{+}$human glioma stem cells effectively enhanced the rate of inhibition of cell proliferation in vitro (Fig. 3B). The qRT-PCR results showed that compared 

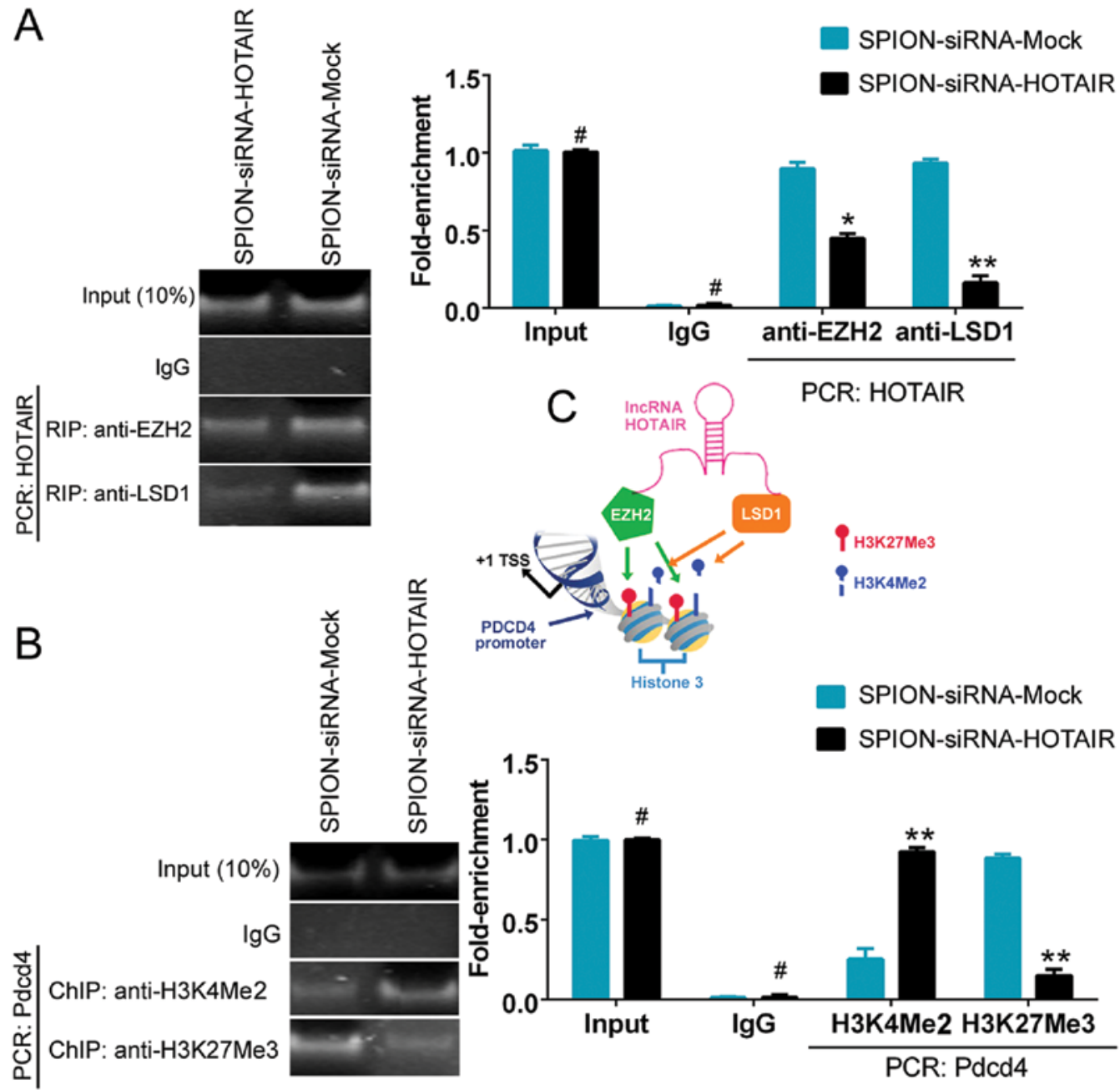

SPION-SIRNA-HOTAIR
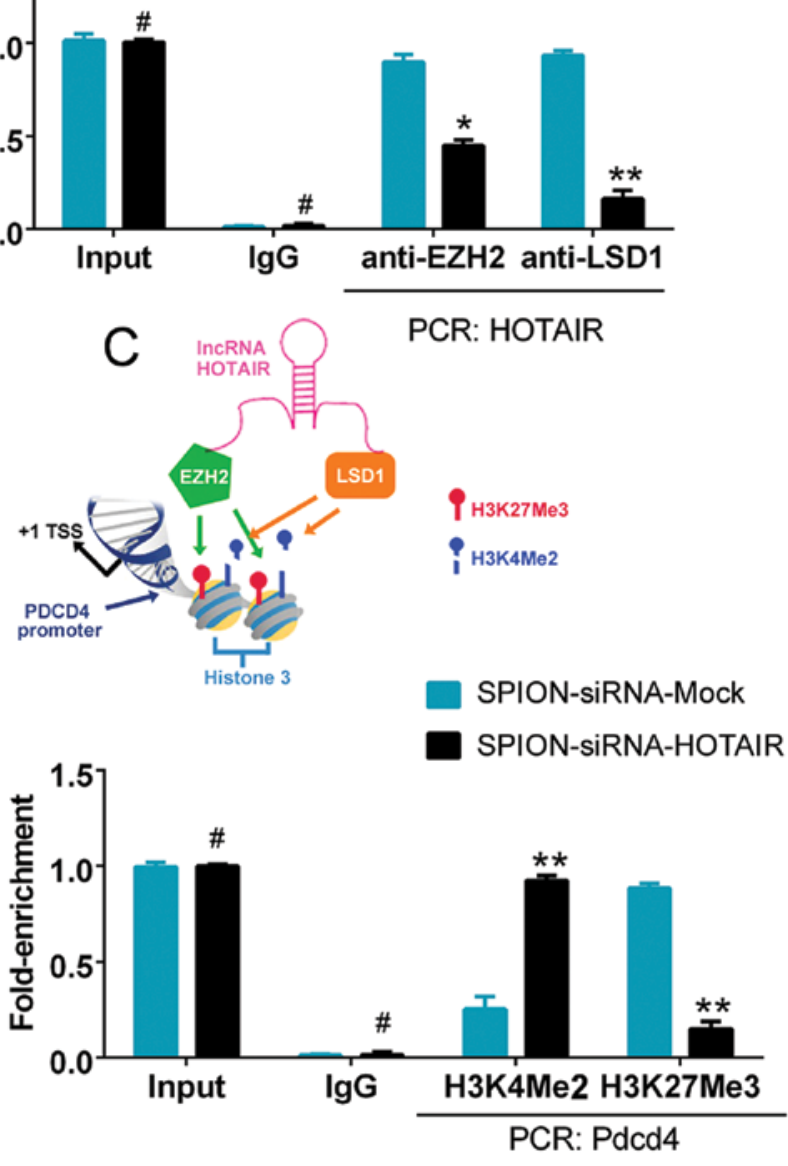

Figure 4. Downregulation of HOTAIR expression reduces the recruitment of the EZH2 and LSD1 proteins, thereby regulating the transcription of PDCD4 (A) RIP results indicated that the binding of HOTAIR fragments to EZH2 and LSD1 was significantly decreased in SPION-mediated si-HOTAIR transfection $\mathrm{CD}_{133^{+}}$human glioma stem cells compared with the control group. IgGs from the corresponding species were used as negative controls. (B) The results of the CHIP assay showed that compared with the control group, there was an elevated level of H3K4me2 and a decreased level of $\mathrm{H} 3 \mathrm{~K} 27 \mathrm{me} 3$ in the promoter region of PDCD4 in the group of $\mathrm{CD} 133^{+}$human glioma stem cells subjected to SPION-mediated si-HOTAIR transfection. (C) The mechanisms by which HOTAIR transcriptionally regulates PDCD4 are as follows: the EZH2 methyltransferase binds to the 5' terminus of HOTAIR and mediates the trimethylation of H3K27 located in the promoter region of PDCD4; LSD1 binds to the 3' terminus of HOTAIR and mediates the demethylation of H3K4me2 in the promoter region of PDCD4; and the combined effects of EZH2 and LSD1 silence the transcription of the PDCD4 gene. ${ }^{*} \mathrm{P}<0.05,{ }^{* *} \mathrm{P}<0.01,{ }^{*} \mathrm{P}>0.05, \mathrm{n}=3$.

with the control group, the expression of PDCD4 was significantly increased, while the expression of BDNF (which is related to cell proliferation) and the cell cycle regulatory factors cyclin D1 (CCND1) and cyclin-dependent kinase 4 (CDK4) was markedly reduced in SPION-si-HOTAIR-transfected glioma stem cells expressing a low level of HOTAIR (Fig. 3C). Moreover, the results of western blot analysis also demonstrated that compared with the control group, a low level of HOTAIR expression led to significantly increased PDCD4 expression and dramatically reduced levels of the BDNF, CCND1 and CDK4 proteins in human glioma stem cells. The above results demonstrated that SPION-mediated low HOTAIR expression in human glioma stem cells significantly promoted the expression of PDCD4 and inhibited the expression of cell cycle regulatory factors.

HOTAIR regulates the expression of $\mathrm{PDCD} 4$ at the transcriptional level by recruiting EZH2 and LSD1. The ability of HOTAIR to recruit and bind to the transcriptional corepres- sors EZH2 and LSD1 was assessed using the RNA-binding protein immunoprecipitation (RIP) method. Fig. 4A shows that the ability of HOTAIR to bind to EZH2 and LSD1 was significantly reduced in the si-HOTAIR-transfected group compared with the control group. The results indicated that the low level of HOTAIR expression affected the recruitment of the EZH2 and LSD1 proteins. To further investigate the effect of HOTAIR on PDCD4, a ChIP assay was conducted. A decreased level of H3K27me3 and an elevated level of $\mathrm{H} 3 \mathrm{~K} 4 \mathrm{me} 2$ were detected in the promoter region of PDCD4 after interference with the expression of HOTAIR. The results indicated that si-HOTAIR regulated PDCD4 promoter-binding histones and, consequently, maintained the transcriptional activation of the PDCD4 gene by affecting HOTAIR expression (Fig. 4B and C).

A low level of HOTAIR expression suppresses tumorigenicity of $\mathrm{CD} 133^{+}$human glioma stem cells in nude mice. Human glioma stem cells that were transfected with SPION/ 

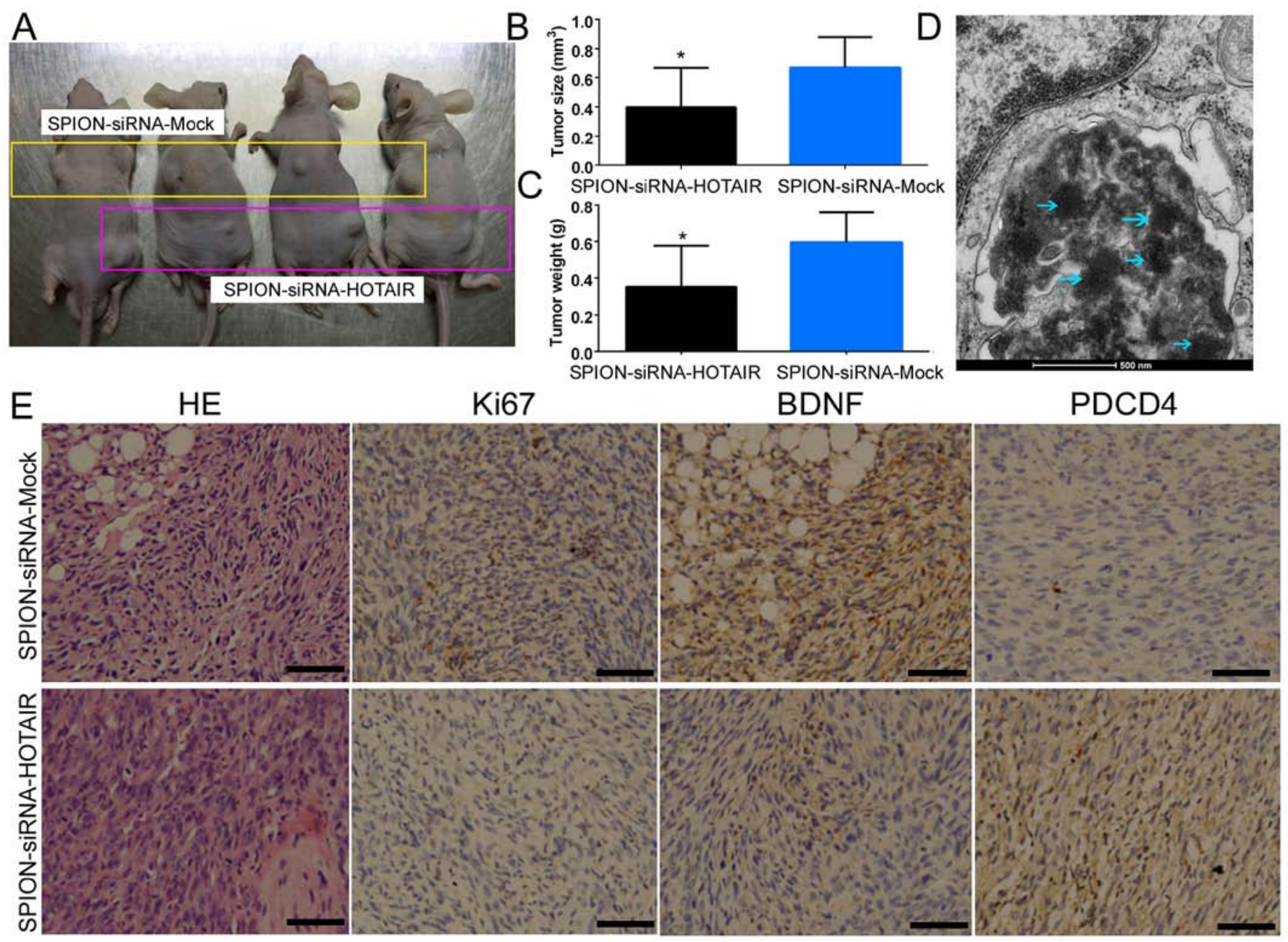

Figure 5. Downregulation of HOTAIR expression significantly inhibits the tumorigenic capacity of CD133+ human glioma stem cells. (A) Si-mock- and si-HOTAIR-transfected $\mathrm{CD}_{133^{+}}$human glioma stem cells formed subcutaneous xenograft tumors in nude mice. The yellow box and red box indicate the respective locations of these tumors. (B) Compared with the control group, CD133+ human glioma stem cells expressing a low level of HOTAIR produced significantly smaller subcutaneous xenograft tumors in nude mice. (C) The weight of the subcutaneous xenograft tumors derived from $\mathrm{CD}_{133^{+}}$human glioma stem cells expressing a low level of HOTAIR was significantly lower compared with the control group. (D) TEM results showed that multiple circular-shaped, high-density electron clouds with the dimension of $80-100 \mathrm{~nm}$ were present in xenograft tumors derived from CD133 ${ }^{+}$human glioma stem cells expressing a low level of HOTAIR. The substance corresponding to the electron clouds was SPION. (E) H\&E staining showed that the two groups of cells were both capable of forming xenograft tumors equivalent to human glioma. However, xenograft tumors derived from si-HOTAIR-transfected glioma stem cells exhibited a significantly lower degree of malignancy compared with those derived from si-mock-transfected glioma stem cells. The results of immunohistochemical analysis showed that the expression of PDCD4 was significantly higher, while the expression of BDNF and Ki67 was significantly lower in xenograft tumors derived from si-HOTAIR-transfected glioma stem cells compared with tumors derived from si-mock-transfected glioma stem cells; x200 (scale bar, $20 \mu \mathrm{m}$ ). ${ }^{*} \mathrm{P}<0.05, \mathrm{n}=4$.

si-HOTAIR or SPION/si-mock conjugates were inoculated subcutaneously into BABL/C ${ }^{\text {nu/nu }}$ mice (Fig. 5A). Statistical analysis showed that human glioma stem cells expressing a low level of HOTAIR exhibited a dramatically reduced tumorigenic rate and produced significantly smaller xenograft tumors compared with the control group (Fig. 5B). Moreover, the tumor weight was significantly reduced in the group of mice inoculated with human glioma stem cells expressing a low level of HOTAIR in comparison with the control group (Fig. 5C). In addition, multiple circular-shaped high-density electron clouds of $\sim 80-100 \mathrm{~nm}$ in size were observed in the xenograft tumor tissues derived from the si-HOTAIR and si-mock-transfected groups (Fig. 5D). It was speculated that the (intercellular) substance corresponding to the electron clouds was SPION. The results of H\&E staining indicated that both groups of cells were capable of generating human glioma xeno- grafts in nude mice. However, xenograft tumors derived from glioma stem cells expressing a low level of HOTAIR exhibited a significantly lower degree of malignancy compared with the control group (Fig. 5E). The results of immunohistochemical staining also indicated that the expression of PDCD4 was significantly increased in the si-HOTAIR-transfected group, while the expression of BDNF and Ki67 (proteins capable of reflecting the proliferative status of the cells) was dramatically decreased (Fig. 5E). The above results indicated that low HOTAIR expression inhibited the growth and tumorigenic capability of $\mathrm{CD}_{133^{+}}$human glioma stem cells in nude mice.

\section{Discussion}

This study showed that inhibition of HOTAIR expression targeted by SPION-mediated siRNA transfection promoted 
the expression of PDCD4 at the transcriptional level, thereby reducing the proliferation, invasion and tumorigenicity of human glioma stem cells.

PDCD4 has been widely recognized as a tumor suppressor gene and a target for antitumor therapy. It is closely related to the development, progression and prognosis of tumors (16). It has been demonstrated that multiple signaling pathways are involved in the regulation of PDCD4 expression. For example, PDCD4 is a downstream target of microRNA 21 (miR21). Inhibition of miR21 in tumor cells increases the expression of endogenous PDCD4 and promotes the apoptosis of tumor cells (17). The relationship between transforming growth factor- $\beta$ (TGF- $\beta$ ) and PDCD4 has not been clearly defined, although it has been found that treatment of the human hepatoma cell line Huh7 with TGF- $\beta$ increases the expression of the PDCD4 gene. However, it has also been shown that TGF- $\beta$ downregulates the expression of PDCD4 through inducing the expression of miR-21 $(18,19)$. In addition to regulating PDCD4 protein expression at the translational level, Akt and s6 kinase 1 (S6K1) induce the phosphorylation of the PDCD4 protein and consequently promote PDCD4 degradation through the ubiquitin-proteasome pathway, thereby decreasing the expression of PDCD4. Drugs targeting the above pathway enhance the level of PDCD4 (16). A study conducted at the transcriptional level demonstrated that the transcription factor v-Myb and DNA demethylation induce the expression of PDCD4 (16). This study revealed, for the first time, that HOTAIR was capable of affecting the expression of PDCD4 through regulating the methylation levels of histone $\mathrm{H} 3 \mathrm{~K} 4$ and H3K27. Our findings showed that inhibition of HOTAIR expression by si-HOTAIR significantly enhanced the mRNA and protein levels of PDCD4.

Histone methylation is a type of epigenetic modification involving the methylation of arginine or lysine residues in the N-terminal domain of histones H3 and H4. The effects of histone methylation are mainly reflected in heterochromatin formation, gene imprinting, $\mathrm{X}$-chromosome inactivation and transcriptional regulation (20). To date, 24 histone methylation sites (mainly at lysines) have been discovered. Histone methylation can occur in the form of mono-, di-, or trimethylation, which is catalyzed by histone methyltransferase and histone demethylase. Methylation of different lysine residues may produce different effects on gene transcription. Methylation at $\mathrm{H} 3 \mathrm{~K} 4, \mathrm{H} 3 \mathrm{~K} 36$ and $\mathrm{H} 3 \mathrm{~K} 79$ activates gene transcription, whereas methylation at $\mathrm{H} 3 \mathrm{~K} 9, \mathrm{H} 3 \mathrm{~K} 27, \mathrm{H} 3 \mathrm{~K} 79$ and $\mathrm{H} 4 \mathrm{~K} 20$ inhibits gene transcription (21). A previous study by our group on renal cancer cells showed that HOTAIR affects the expression of the cell cycle-related proteins p53, p21 and p16 at the transcriptional level through binding to EZH2 and histone $\mathrm{H} 3$ trimethyl Lys27 (H3K27me3) (9). This study further investigated the mechanisms by which HOTAIR regulates PDCD4. It was found that HOTAIR recruited and enriched the EZH2 and LSD1 proteins. EZH2 maintained the trimethylation state of histone $\mathrm{H} 3 \mathrm{~K} 27$ in the promoter region of PDCD4, while LSD1 functioned as a histone deacetylase to mediate the demethylation of H3K4me2. The synergistic effect of EZH2 and LSD1 led to the inhibition of PDCD4 transcription. Inhibition of HOTAIR expression by si-HOTAIR reduced the recruitment of the EZH2 and LSD1 proteins, thereby upregulating the expression of PDCD4 at the epigenetic level.
Taking into account the phospholipid bilayer structure of the eukaryotic cell membrane, past studies have generally employed lipid carriers as the medium for plasmid DNA transfection. Although liposomes have been used for many years, the transfection efficiencies of liposomes are not particularly high. With the development of nanotechnology research, certain studies have reported the use of nanoparticles as transfection media for intracellular delivery of drugs or nucleic acids. In this study, SPIONs were employed as a transfection vehicle to deliver siRNA into cells. The transfected cells were analyzed via TEM, and SPION signals were detected in the transfected cells. Northern blot analysis showed that the HOTAIR hybridization signal was significantly reduced in si-HOTAIR-transfected human glioma stem cells. The results indicated that SPION successfully facilitated the passage of si-HOTAIR through the cell membrane and interfered with the amplification of the target genes. Both in vivo and in vitro experiments demonstrated that the in vivo and in vitro proliferation and invasion of glioma cells were inhibited, and the tumorigenic capacity of glioma cells was significantly reduced after suppressing the expression of HOTAIR by the method described above.

In conclusion, SPIONs exhibited great potential in mediating the transfection of nucleic acids into mammalian cells. SPIONs effectively mediated the expression of si-HOTAIR in human glioma stem cells. Si-HOTAIR inhibited the downstream expression of PDCD4 at the transcriptional level and ultimately suppressed the proliferation, invasion and tumorigenicity of glioma stem cells.

\section{Acknowledgements}

This study was supported by grants from National Natural Science Foundation of China (no. 81202811, 81371410), and Project funded by China Postdoctoral Science Foundation (no. 2014M550250, 2015T80455), and Shanghai Natural Science Foundation (no. 16ZR1434000) to Te Liu. It was also partially supported by the Biomedical Multidisciplinary Program of Shanghai Jiao Tong University (no. YG2014MS31) to Yuncheng $\mathrm{Wu}$.

\section{References}

1. Reya T, Morrison SJ, Clarke MF and Weissman IL: Stem cells, cancer, and cancer stem cells. Nature 414: 105-111, 2001.

2. He J, Liu Y and Lubman DM: Targeting glioblastoma stem cells: Cell surface markers. Curr Med Chem 19: 6050-6055, 2012.

3. Galli R, Binda E, Orfanelli U, Cipelletti B, Gritti A, De Vitis S, Fiocco R, Foroni C, Dimeco F and Vescovi A: Isolation and characterization of tumorigenic, stem-like neural precursors from human glioblastoma. Cancer Res 64: 7011-7021, 2004.

4. Kapranov P, Cheng J, Dike S, Nix DA, Duttagupta R, Willingham AT, Stadler PF, Hertel J, Hackermüller J, Hofacker IL, et al: RNA maps reveal new RNA classes and a possible function for pervasive transcription. Science 316: 1484-1488, 2007.

5. Rinn JL, Kertesz M, Wang JK, Squazzo SL, Xu X, Brugmann SA, Goodnough LH, Helms JA, Farnham PJ, Segal $\mathrm{E}$, et al: Functional demarcation of active and silent chromatin domains in human HOX loci by noncoding RNAs. Cell 129: 1311-1323, 2007.

6. Shah N and Sukumar S: The Hox genes and their roles in oncogenesis. Nat Rev Cancer 10: 361-371, 2010.

7. Tsai MC, Manor O, Wan Y, Mosammaparast N, Wang JK, Lan F, Shi Y, Segal E and Chang HY: Long noncoding RNA as modular scaffold of histone modification complexes. Science 329: 689-693, 2010 . 
8. Hayami S, Kelly JD, Cho HS, Yoshimatsu M, Unoki M, TsunodaT, Field HI, Neal DE, Yamaue H, Ponder BA, et al: Overexpression of LSD1 contributes to human carcinogenesis through chromatin regulation in various cancers. Int J Cancer 128: 574-586, 2011.

9. Wu Y, Liu J, Zheng Y, You L, Kuang D and Liu T: Suppressed expression of long non-coding RNA HOTAIR inhibits proliferation and tumourigenicity of renal carcinoma cells. Tumour Biol: 35: 11887-11894, 2014.

10. Zhou X, Ren Y, Zhang J, Zhang C, Zhang K, Han L, Kong L, Wei J, Chen L, Yang J, et al: HOTAIR is a therapeutic target in glioblastoma. Oncotarget 6: 8353-8365, 2015.

11. Wang Y, Cui H, Li K, Sun C, Du W, Cui J, Zhao X and Chen W: A magnetic nanoparticle-based multiple-gene delivery system for transfection of porcine kidney cells. PLoS One 9: e102886, 2014.

12. Delyagina E, Schade A, Scharfenberg D, Skorska A, Lux C, Li W and Steinhoff G: Improved transfection in human mesenchymal stem cells: Effective intracellular release of pDNA by magnetic polyplexes. Nanomedicine (Lond) 9: 999-1017, 2014.

13. Wang Y, Cui H, Sun C, Du W, Cui J and Zhao X: Study on performance of magnetic fluorescent nanoparticles as gene carrier and location in pig kidney cells. Nanoscale Res Lett 8: 127, 2013.

14. Gao Y, Liu T and Huang Y: MicroRNA-134 suppresses endometrial cancer stem cells by targeting POGLUT1 and Notch pathway proteins. FEBS Lett 589: 207-214, 2015.

15. Cheng W, Liu T, Wan X, Gao Y and Wang H: MicroRNA-199a targets CD44 to suppress the tumorigenicity and multidrug resistance of ovarian cancer-initiating cells. FEBS J 279: 2047-2059, 2012 .
16. Lankat-Buttgereit B and Göke R: The tumour suppressor Pdcd4: Recent advances in the elucidation of function and regulation. Biol Cell 101: 309-317, 2009.

17. Poria DK, Guha A, Nandi I and Ray PS: RNA-binding protein HuR sequesters microRNA-21 to prevent translation repression of proinflammatory tumor suppressor gene programmed cell death 4. Oncogene 35: 1703-1715, 2016.

18. Yao Q, Cao S, Li C, Mengesha A, Kong B and Wei M: Micro-RNA-21 regulates TGF-beta-induced myofibroblast differentiation by targeting PDCD4 in tumor-stroma interaction. Int J Cancer 128: 1783-1792, 2011.

19. Zhang H, Ozaki I, Mizuta T, Hamajima H, Yasutake T, Eguchi Y, Ideguchi $\mathrm{H}$, Yamamoto $\mathrm{K}$ and Matsuhashi S: Involvement of programmed cell death 4 in transforming growth factor-beta1induced apoptosis in human hepatocellular carcinoma. Oncogene 25: 6101-6112, 2006.

20. Ning B, Li W, Zhao W and Wang R: Targeting epigenetic regulations in cancer. Acta Biochim Biophys Sin (Shanghai) 48: 97-109, 2016.

21. Barski A, Cuddapah S, Cui K, Roh TY, Schones DE, Wang Z, Wei G, Chepelev I and Zhao K: High-resolution profiling of histone methylations in the human genome. Cell 129: 823-837, 2007. 